\title{
Biomechanics of the Intervertebral Disc in Compression: Experimental and Numerical Study
}

\author{
Ramiro Arturo González Gutiérrez, Vladimir Rangel Alonso and José Gustavo Zambrano Rosas \\ Department of Metalworking, Instituto Tecnológico de Tijuana, Tijuana 22414, Mexico
}

Received: November 04, 2014 / Accepted: November 17, 2014 / Published: January 25, 2015.

\begin{abstract}
Loading history and age are factors for disc degeneration and disc biomechanics; however, their relationship is unclear. To evaluate disc biomechanics, we conducted an experimental, anatomical and numerical approach to distinguish discs with mild and severe degeneration. In the experimental procedure, 10 cadaveric lumbar discs are tested to static and dynamic compression, and the elastic and viscous moduli and the dynamic parameters are reported. The morphology of disc degeneration is gained with MRI (magnetic resonance imaging) and used to generate a nonlinear finite element model of a degenerated disc, and assisted with the experimental results in order to numerically investigate the distribution of stresses and strains within the disc. The results show a promising methodology for the study of intervertebral disc biomechanics and in general other tissues, organs and medical devices.
\end{abstract}

Key words: Intervertebral disc, viscoelasticity, testing, magnetic resonance imaging, finite element model.

\section{Introduction}

The intervertebral disc is a fibrocartilage structure composed of a nucleus pulpous, annulus fibrosis, and cartilage endplate (Fig. 1). The disc along with the facet joints carries all the loading to which the trunk is subjected. This includes compression, shear, bending and torsion, acting together statically or dynamically [1].

The biomechanics of disc loading changes with age and degeneration [1], and its study have been the subject of numerous investigations: experimentally, numerically, and clinically [2-5]. Testing discs in compression serves to evaluate disc stiffness, Young's modulus, and storage and loss moduli [2-4]. From a viscoelastic approach, degenerated discs have shown a reduction in disc stiffness, movement, stress relaxation and damping capacity [5]. Such results represent valuable data as inputs into numerical models, such as the FEM (finite element method), for

Corresponding author: Ramiro Arturo González Gutiérrez, professor, Ph.D., research fields: mechanical design, biomechanics, finite element modeling. E-mail: ramiro.gonzalez@tectijuana.edu.mx.

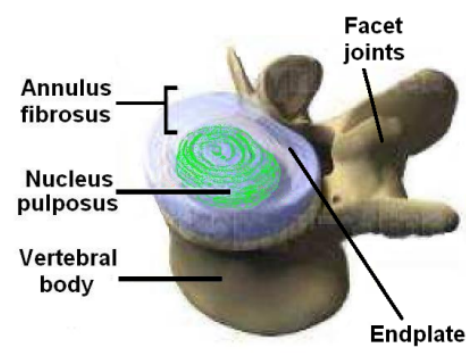

Fig. 1 Intervertebral disc components.

investigating mechanical properties of the disc tissues and the distribution of stress and strain inside the disc. Thus, we developed one FEM of a degenerated disc based on magnetic resonance imaging and investigated the stress and strain distribution using also testing results from a group of degenerated discs. The paper is organized as follows: Sections 2-5 give the materials and methodologies; Sections 6-8 present the results from the MRI (magnetic resonance imaging) inspection, testing and the FEM simulation, respectively; Section 9 addresses the implications of the results; and Section 10 gives the main conclusions.

\section{The Specimens}

Ten lumbar intervertebral discs from elderly donors 
obtained from Hospital Clinic of Barcelona, Spain were used in this study. After dissection, all specimens were examined to verify absence of any disease, and measurements of the disc height and cross section were recorded before the inspection with MRI. The anatomical data are given in Table 1.

\section{MRI Inspection}

A Siemens Harmony ${ }^{\mathrm{TM}}$ system was used with a spin-echo $T_{1}$ and echo-gradient $T_{2}$ sequences in accordance with Ref. [6]. The tomography resolution for all discs were $256 \times 256$ pixels, field of view of $10.4 \mathrm{~mm} \times 10.4 \mathrm{~mm}$; slide thickness of $0.4 \mathrm{~mm}$, and slide interspace of $0.4 \mathrm{~mm}$. One tomography was used for developing the FEM of disc degeneration, using additionally MIMICSTM (MATERIALISE ${ }^{\mathrm{TM}}$, Leuven, Belgium) and MARC MENTAT ${ }^{\mathrm{TM}}$ (MSC Software ${ }^{\mathrm{TM}}$, Santa Ana CA, USA) software for treatment of medical imaging, and stress and strain analyses, respectively. The procedure used in this study is outlined in Fig. 2.

\section{Experimental Protocol}

Biomechanical testing to all intervertebral discs was done at the biomaterials laboratory of the CMEM

Table 1 Mean and standard deviation of the disc age, size and height.

\begin{tabular}{llllll}
\hline Discs & $\begin{array}{l}\text { Lumbar } \\
\text { level }\end{array}$ & $\begin{array}{l}\text { Sex } \\
\mathrm{M}=\text { Female }\end{array}$ & $\begin{array}{l}\text { Age } \\
\text { (years) }\end{array}$ & $\begin{array}{l}\text { Cross } \\
\text { section } \\
\left(\mathrm{mm}^{2}\right)\end{array}$ & $\begin{array}{l}\text { Height } \\
(\mathrm{mm})\end{array}$ \\
\hline$n=5$ & $\mathrm{~L} 23$ & $\mathrm{~F}=1, \mathrm{M}=4$ & $70(3.5)$ & $\begin{array}{l}1,739 \\
(184)\end{array}$ & $9.8(1.8)$ \\
$n=5$ & $\mathrm{~L} 45$ & $\mathrm{~F}=1, \mathrm{M}=4$ & $70(3.5)$ & $\begin{array}{l}1,951 \\
(223)\end{array}$ & $10(1.6)$ \\
\hline
\end{tabular}

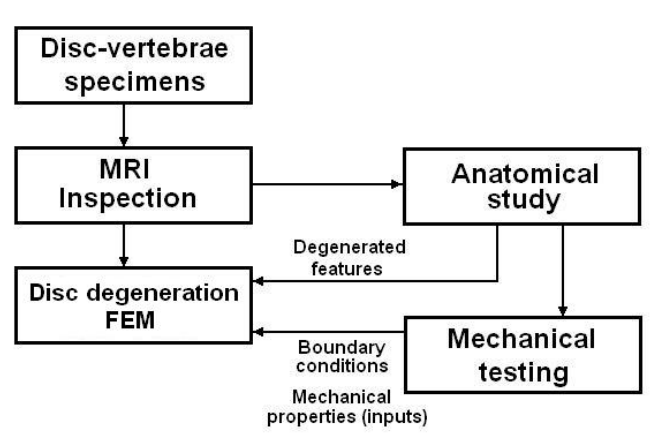

Fig. 2 Procedure used.
(Departament of Materials Science and Metallurgy) of the Polytechnic University of Catalonia. The experimental protocol was based on previous studies $[2,4]$ and included axial compression and dynamic compression. The compressive modulus and stress relaxation were determined using static loading and adjusting data to a Kelvin-Voigt model as follows:

$$
\begin{gathered}
\sigma(t)=E \varepsilon(t)+\eta \frac{\mathrm{d} \varepsilon(t)}{\mathrm{d} t} \\
E=\frac{\sigma_{0}}{\varepsilon_{0}} \\
E_{R}(t)=\frac{\sigma_{0}}{\varepsilon_{0}} \exp ^{-\left(\frac{E}{\eta}\right) t} \\
E_{R}(t)=\frac{\sigma_{0}}{\varepsilon_{0}} \exp ^{-\left(\frac{t}{t_{R}}\right)}
\end{gathered}
$$

where, $E$ is the compressive modulus, $\varepsilon(t)$ is time dependent strain, $\eta$ is viscosity, $\sigma_{0}$ is stress, $\varepsilon_{0}$ is strain, $E_{R}$ is the relaxation modulus, $t_{R}$ is time decay from $\sigma_{0}$ to a stress equal to $(1 / \mathrm{exp})^{*} \sigma_{0}$.

The disc storage $E^{\prime}$ and loss $E^{\prime \prime}$ moduli and hysteresis $H$ were determined using cyclic compression and adjusting data as follows:

$$
\begin{gathered}
E^{\prime}=\frac{\sigma_{0}}{\varepsilon_{0}} \cos \beta \\
E^{\prime \prime}=\frac{\sigma_{0}}{\varepsilon_{0}} \sin \beta \\
\tan \delta=\frac{E^{\prime \prime}}{E^{\prime}} \\
H=\left[\int_{r_{1}}^{r_{2}} \sigma \mathrm{d} \varepsilon\right]_{\text {load }}-\left[\int_{r_{1}}^{r_{2}} \sigma \mathrm{d} \varepsilon\right]_{\text {unload }}
\end{gathered}
$$

where, $\beta$ is the phase shift between stress and strain, and $\tan \delta$ and $H$ represents the ratio of storage to loss moduli and the hysteresis, respectively.

\section{Numerical Protocol}

Thresholding of the disc and vertebrae was the first step in creating the FEM. This task included creation of masks for the various segmentations through Boolean algebra, and identification of cortical and cancellous bone from the vertebrae, and nucleus, annulus, endplate and cavities from the disc. The 
procedure in MIMICS ${ }^{\mathrm{TM}}$ is outlined in Fig. 3, and a $3 \mathrm{D}$ view of the regions of segmentation is shown in Fig. 4.

The finite element mesh was created in MARCTM and was based on tetrahedral element \# 157 with four nodes, ideally suitable for incompressible materials. Five models were developed base on the triangle size: $1 \mathrm{~mm}, 1.5 \mathrm{~mm}, 2 \mathrm{~mm}, 3 \mathrm{~mm}$ and $5 \mathrm{~mm}$, which were in accordance with the size of most degenerative features. Material formulation included orthotrophy for cancellous and cortical bone, with values of the Young's moduli in the range of $140 \mathrm{MPa}<E<22,000 \mathrm{MPa}$, Shear modulus $48 \mathrm{MPa}<G<5,400 \mathrm{MPa}$, Poisson ratio 0.20 $<v<0.48$; disc materials were treated as incompressible and elastomeric with a formulation based on the Mooney-Rivlin solid model [7, 8]:

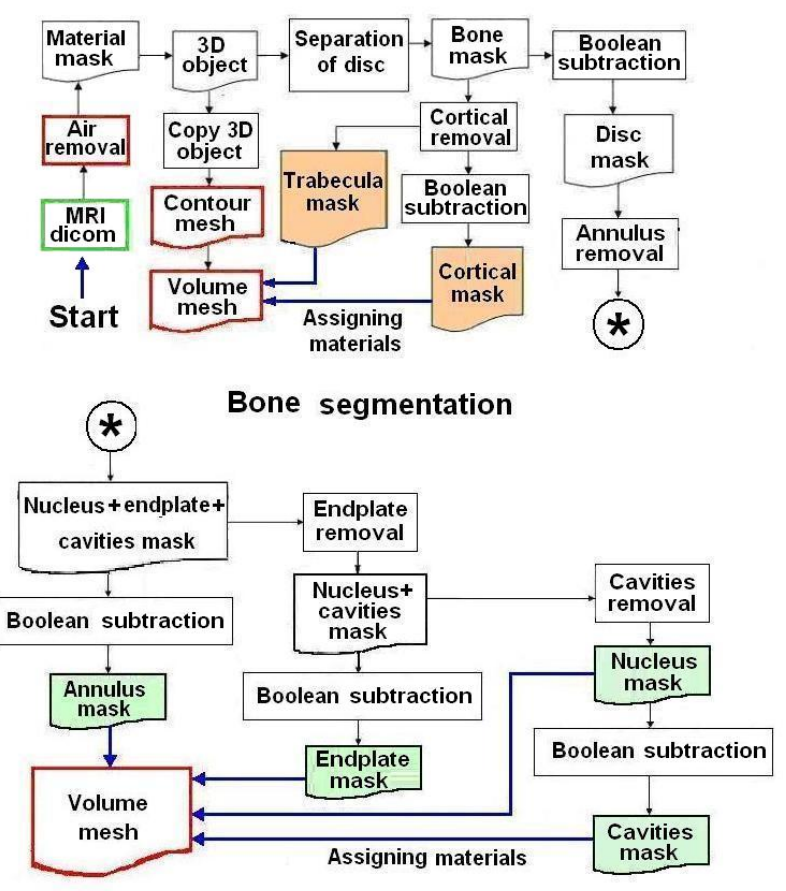

Disc segmentation

Fig. 3 Segmentation procedure for bone and disc.
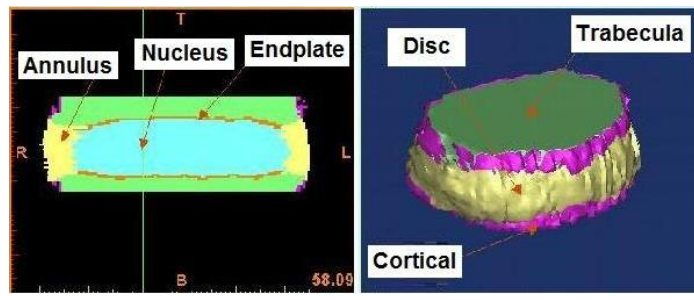

Fig. 4 Disc model showing segmentation.

$$
W=\mathrm{C}_{1}\left(I_{1}-3\right)+\mathrm{C}_{2}\left(I_{2}-3\right)
$$

where, $I_{1}$ and $I_{2}$ are the first and second invariant of the Cauchy strain tensor, $\mathrm{C}_{1}$ and $\mathrm{C}_{2}$ are empiric constants of the material with values shown in Table 2 .

The constants for the annulus fibrosus were kept fix $\left(C_{1}=0.10\right.$ and $\left.C_{2}=0.025\right)$ and only the constants for the nucleus pulposus were adjusted, on the basis that under compression, the main deformation occurs in the nucleus [9]. The criterion used for the adjustment of the Mooney-Rivlin constants were based on the reaction F.D. (force difference) between those from the simulation $\left(F_{S}\right)$ and the testing $(1,000 \mathrm{~N})$, as follows:

$$
F . D .=\sqrt{\left(F_{S}-1,000\right)^{2}}
$$

All disc models were submitted to the same boundary conditions corresponding to the compression testing. The type of analysis used was a nonlinear static and the displacements, reaction force, stress and strain for the intervertebral disc and vertebrae materials were investigated.

\section{Degenerative Features with MRI}

The MRI inspection revealed that the lumbar level was sensitive with degeneration scoring (student T-test, $P<0.05)$. The main degenerative features identified in the intervertebral discs are shown in Fig. 5.

In general, cavity formation appeared in the nucleus region and in the annulus of all discs to a lesser extent. However, the L45 discs showed more cavities with a clear collapse of the disc height, abundance of schmorol's nodes in the disc-vertebrae boundary, bone reactive sclerosis and bone formation at the annulus anterior and posterior sides. Thus, L23 discs with maintenance of the disc height were assigned a mild

Table 2 Mooney-Rivlin $\mathrm{C}_{1}$ and $\mathrm{C}_{2}$ constants [8].

\begin{tabular}{lll}
\hline Material & Formulation & $\mathrm{C}_{1}$ and $\mathrm{C}_{2}$ value range \\
\hline Annuls & Elastomeric & $0.10<\mathrm{C}_{1}<0.20$ \\
Fibrosis & Mooney-Rivlin & $0.025<\mathrm{C}_{2}<0.05$ \\
Nucleus & Elastomeric & $0.03<\mathrm{C}_{1}<0.12$ \\
pulpous & Mooney-Rivlin & $0.01<\mathrm{C}_{2}<0.03$ \\
Endplate & Isotropic & $E=20 \mathrm{MPa}, v=0.30$ \\
\hline
\end{tabular}




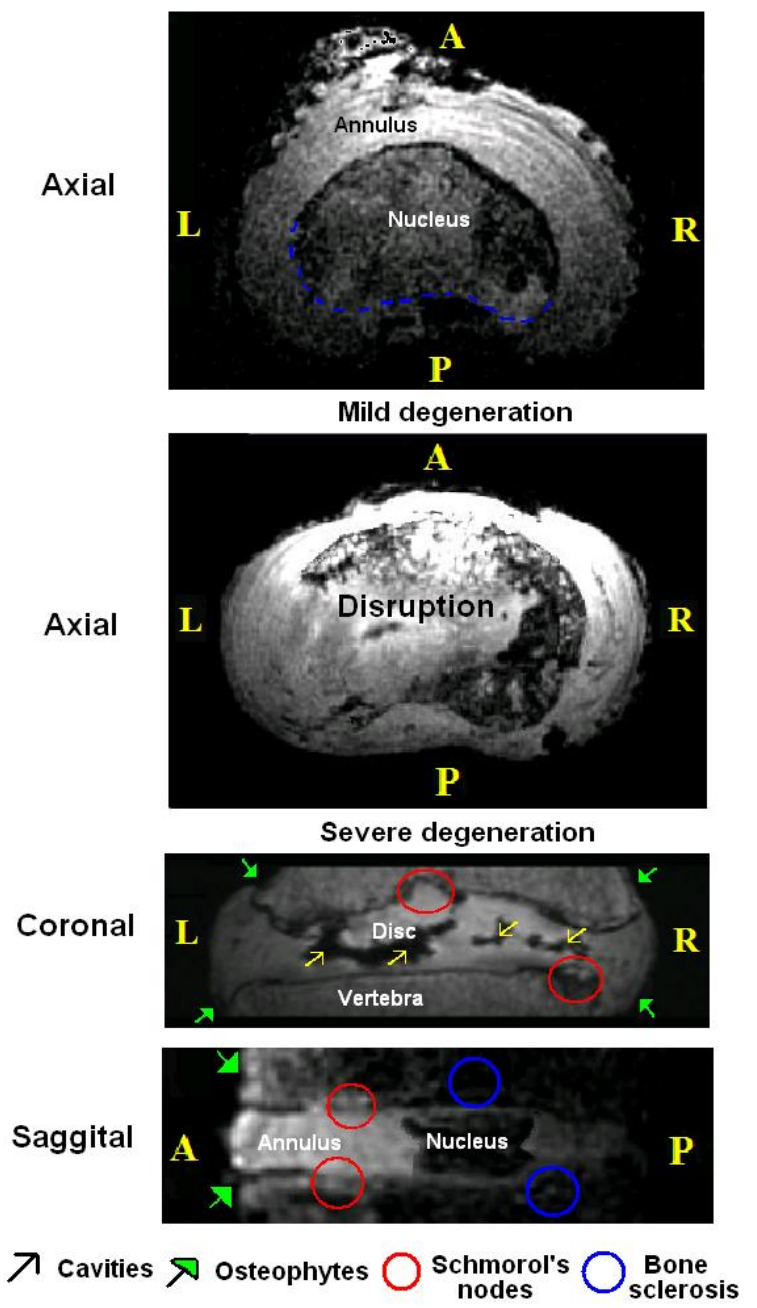

Fig. 5 Degenerative features for mild and severe scoring.

degeneration scoring, and discs with a collapse of the disc space typically from the lower L45 level were assigned a severe degeneration scoring.

\section{Disc under Static and Dynamic Compression}

Under static and dynamic loading, there were no significant differences among mechanical properties and lumbar level (student T-test, $P>0.05$ ) due to the large scattering. However, under static compression, the discs showed a nonlinear stress-strain relationship with large deformations axially and radially. Stress relaxation was faster in highly degenerated discs which showed a lower relaxation modulus (Table 3 ).

Increase in axial stiffness and dissipation was observed with increase in frequency loading. Storage modulus $E$ ' decreases up to $20 \%$ less in discs with severe degeneration, although the loss modulus was about even between degeneration scorings. In general, the mean storage modulus $E$ ' was around 20 times larger than the mean loss modulus $E$ " (Table 4).

\section{FEM of Disc Degeneration and Compression Simulation}

Based on the convergence test, the most convenient model was that of an element size of $1.5 \mathrm{~mm}$. The selected model included 187,746 elements and 43,172 nodes spread in six regional material masks. Next, the initial selection of the Mooney-Rivlin constants for the nucleus pulpous were $C_{1}=0.12$ and $C_{2}=0.03$, these values were modified as a function of the reaction force. A $1 \%$ difference in F.D. was used as the tolerance. The resulting values were $\mathrm{C}_{1}=0.07$ and $\mathrm{C}_{2}=0.02$. The algorithm for the adjustment of the Mooney-Rivlin constants is shown in Fig. 6.

The strain distribution showed that the nucleus bears most of the highest deformation, reaching $-55 \%$, the annulus distribution showed strains from $+10 \%$ to $-40 \%$. In contrast, the vertebrae deformed less than $5 \%$, with $0.25 \%$ at cortical region (Fig. 7 ).

Table 3 Mean values and standard deviation for the motion response under static compression.

\begin{tabular}{lll}
\hline \multirow{2}{*}{ Parameter } & \multicolumn{2}{c}{ Degeneration scoring } \\
\cline { 2 - 3 } & Mild & Severe \\
\hline Stiffness (N/mm) & $1,277(622)$ & $1,206(562)$ \\
Deformation (\%) & $9.55(3.44)$ & $10.27(4.27)$ \\
Radial bulging (mm) & $0.33(0.35)$ & $0.87(0.62)$ \\
Young's modulus (MPa) & $14.80(12.30)$ & $10.63(2.80)$ \\
Relaxation time (s) & $1,193(307)$ & $962(293)$ \\
Relaxation modulus (MPa) & $3.80(3.52)$ & $2.36(0.61)$ \\
\hline
\end{tabular}

Table 4 Mean values and standard deviation for the motion response under dynamic compression.

\begin{tabular}{|c|c|c|c|c|}
\hline $\begin{array}{l}\text { Frequency } \\
(\mathrm{Hz})\end{array}$ & $\begin{array}{l}\text { Illness } \\
\text { scoring }\end{array}$ & $\begin{array}{l}E^{\prime} \text { modulus } \\
(\mathrm{MPa})\end{array}$ & $\begin{array}{l}\text { E" modulus } \\
(\mathrm{MPa})\end{array}$ & $\begin{array}{l}\text { Hysteresis } \\
\text { (J) }\end{array}$ \\
\hline \multirow{2}{*}{0.1} & Mild & $4.5(2.5)$ & $0.25(0.22)$ & $0.11(0.08)$ \\
\hline & Severe & $4.0(2.0)$ & $0.25(0.20)$ & $0.11(0.08)$ \\
\hline \multirow{2}{*}{1} & Mild & $4.8(1.9)$ & $0.30(0.25)$ & $1.02(0.87)$ \\
\hline & Severe & $4.5(2.3)$ & $0.75(0.33)$ & $0.60(0.25)$ \\
\hline \multirow{2}{*}{5} & Mild & $8.0(3.4)$ & $2.1(1.1)$ & $3.15(1.50)$ \\
\hline & Severe & $6.5(2.7)$ & $3.0(1.3)$ & $2.70(1.72)$ \\
\hline
\end{tabular}




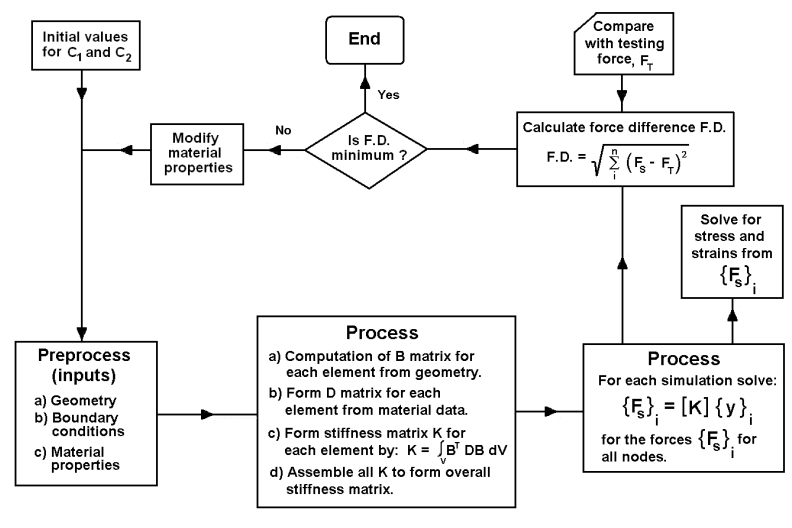

Fig. 6 Algorithm used for adjustment of properties.

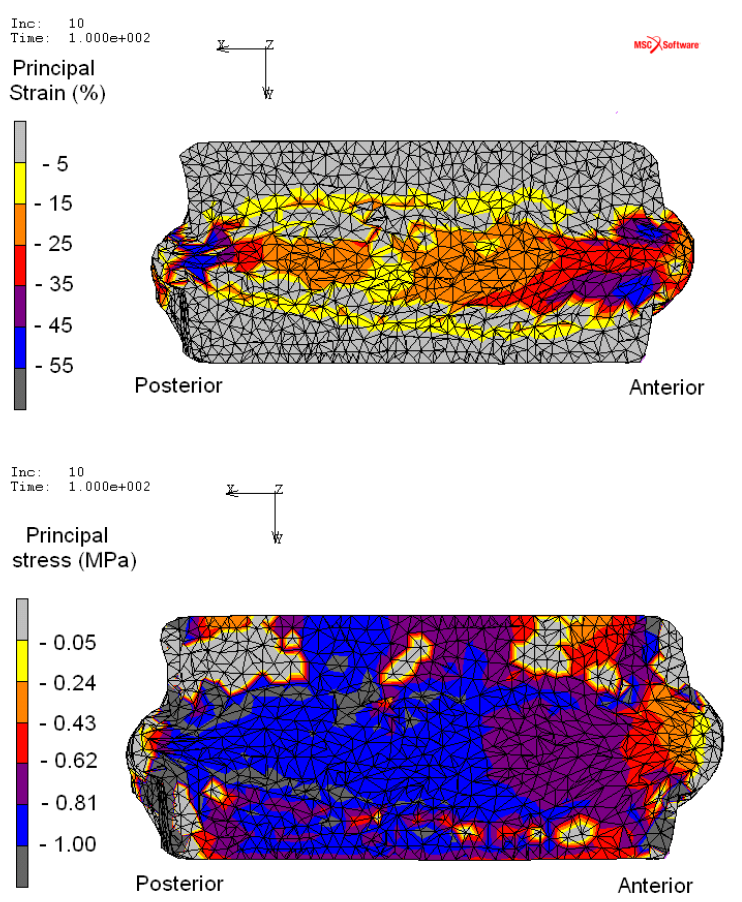

Fig. 7 Stress and strain distributions in the FEM.

The stress distribution showed that the nucleus is subjected to a state of compression, reaching - $1 \mathrm{MPa}$, whereas the annulus is put to tensile and compressive states of stress ranging from $+0.25 \mathrm{MPa}$ to $-1.25 \mathrm{MPa}$ (Fig. 7). The principal stresses in the vertebral regions were from $4 \mathrm{MPa}$ to $-8 \mathrm{MPa}$ in tensile and compression, respectively. The cortical bone was the most stressed part due to its high rigidity.

\section{Discussion}

The MRI findings indicate that discs from the lower lumbar level L45 are more likely to exhibit disc collapse and disruption, which implies a severe loading history in accordance with the clinical experience. The schmorol's nodes and osteophyte formation imply severe degeneration, and with disc disruption limited the identification of the nucleus, annulus and endplate. In contrast, void formation around the nucleus was a reliable indicator of early stages of degeneration, as described elsewhere [10, 11]. Thus, the MRI from the L23 disc was more appropriate for identification of regional materials and developing a FEM of disc degeneration. Segmentation between vertebrae and disc was straightforward, and between annulus, nucleus and endplate depended on contrast.

The biomechanical testing indicates that degeneration diminished their viscoelastic properties, stress relaxation, damping and dissipation capacities, in accordance with previous studies [12-14]. However, the large scatter in the results was normal which caused a poor correlation. In general, individual differences in lumbar disc mechanical behavior usually overshadow any class differences with respect to age, sex, disc level, or degree of degeneration [2].

Other disc models based on medical images have been developed in Refs. [15, 16], but only few have included experimental results. The disc model presented here is suitable for the study of stress and strains. The adjustment of the Mooney-Rivlin constants $\mathrm{C}_{1}$ and $\mathrm{C}_{2}$ for the nucleus correspond to a mild degeneration state which is consistent with the MRI and previous numerical studies [8]. The large deformations in the annulus and nucleus are due to the incompressible and elastomeric formulations, which takes into account the high content of water in the disc. The model also predicts bulging in the annulus and nucleus in accordance with Ref. [17] being larger in the anterior sides, where more water is present. The stress distribution indicates that the nucleus is subjected to a compressive state and suggests that the nucleus is the main carrier of the load as described elsewhere $[8,9]$. 


\section{Conclusions}

The proposed methodology is novel, versatile, functional and economic. Therefore, its application has potential for the medical sciences, clinical field, design and innovation of medical devices, implants, prosthesis and its manufacture.

\section{Acknowledgements}

The authors will like to express their gratitude to the following researchers: M. D. Manel Llusa, M. D. Jaume Pomes, Dr. Josep Planell and Dr. Damien Lacroix and our alma mater Instituto Tecnológico de Tijuana.

\section{References}

[1] Adams, M. A., and Roughley, P. J. 2006. "What Is Intervertebral Disc Degeneration, and What Causes It?" Spine 31: 2151-61.

[2] Nachemson, A., Schultz, A. B., and Berkson, M. H. 1979. "Mechanical Properties of Human Lumbar Spine Motion Segments. Influence of Age, Sex, Disc Level and Degeneration." Spine 4 (1): 1-8.

[3] Schultz, A. B., Berkson, M. H., and Nachemson, A. L. 1979. "Mechanical Properties of Lumbar Human Spine Motion Segments-Part II: Responses in Compression and Shear; Influence of Gross Morphology." J. Biomech. Eng. 101 (1): 53-7.

[4] Kasra, M., Shirazi, A. A., and Drouin, G. 1992. "Dynamics of Human Lumbar Intervertebral Joints: Experimental and Finite Element Investigations." Spine 17 (1): 93-102.

[5] Kazarian, L. E. 1975. "Creep Characteristics the Human Spinal Column.” Orthop. Clin. North A. 6 (1): 3-18.

[6] Brinckmann, P., Frobin, P. W., Kramer, M., and Hartwig, E. 2001. "Height of Lumbar Discs Measured from Radiographs Compared with Degeneration and Height Classified from MR Images." European Radiology 11: 263-9.

[7] Lu, Y. M., Hutton, W. C., and Gharpuray, V. M., 1996. "Do Bending, Twisting, and Diurnal Fluid Changes in the Disc Affect the Propensity to Prolapse? A Viscoelastic
Finite Element Model." Spine 21: 2570-9.

[8] Schmidt H., Kettler A., Heuer, F., Simon, U., Claes, L., and Wilke, H. J. 2007. "Intradiscal Pressure, Shear Strain, and Fiber Strain in the Intervertebral Disc under Combine Loading." Spine 32: 748-55.

[9] Noailly, J., Wilke, H. J., Planell, J., and Lacroix, D. 2007. "How Does the Geometry Affect the Internal Biomechanics of a Lumbar Spine Bi-segment Finite Element Model? Consequences on the Validation Process." Journal of Biomechanics 40 (11): 2414-25.

[10] Thompson, J. P., Pearce, R. H., Schechter, M. T., Adams, M. E., Tsang, I. K., and Bishop, P. B. 1990. "Preliminary Evaluation of a Scheme for Grading the Gross Morphology of the Human Intervertebral Disc." Spine 15 (5): 411-5.

[11] Pfirmann, C., Metzdorf, A., Zanetti, M., Hodler, J., and Boos, N. 2001. "Magnetic Resonance Classification of Lumbar Intervertebral Disc Degeneration." Spine 26 (17): 1873-8.

[12] Keller, T. S., Spengler, D. M., and Hansson, T. H. 1987. "Mechanical Behavior of the Human Lumbar Spine. I. Creep Analysis during Static Compressive Loading." Journal of Orthopedic Research 5 (4): 467-78.

[13] Allen, K., and Athanasiou, K. 2006. "Viscoelastic Characterization of the Porcine Temporomandibular Joint Disc under Unconfined Compression.” J. Biomechanics 39: 312-22.

[14] Tanaka, E., Aoyama, J, Tanaka, M., Murata, H., Hamada, T., and Tanne, K. 2002. "Dynamic Properties of Bovine Temporomandibular Joint Disks Change with Age". Journal of Dentistry Research 81 (9): 618-22.

[15] Li, H., and Wang, Z. 2006. Intervertebral Disc Biomechanical Analysis Using the Finite Element Modeling Based on Medical Images." Computerized Medical Imaging and Graphics 30: 363-70.

[16] Wang, Z., and Li, H. 2005. “A Novel 3D Finite Element Modeling Based on Medical Image for Intervertebral Disc Biomechanical Analysis." Presented at the 27th Annual Conference of the IEEE Engineering in Medicine and Biology, Shanghai, China.

[17] Schmidt, H., Kettler, A., Rohlmann, A., Claes, L., and Wilke, H. J. 2007. "The Risk of Disc Prolapses with Complex Loading in Different Degrees of Disc Degeneration-A Finite Element Analysis." Clinical Biomechanics 22 (9): 988-98. 